\section{Arrangements for on scene medical care at major incidents}

\section{Matthew W Cooke}

Accident and Emergency

Medicine Department,

Dudley Road Hospital,

Birmingham B18 7QH

Matthew W Cooke, senior registrar

BMJ 1992;305:748 two provisions.
The regulations on arrangements for dealing with major incidents, ${ }^{1}$ issued in October 1990, required all listed hospitals to draw up a major incident plan, designate a medical incident officer, and arrange for a mobile medical team. This study investigates the extent to which hospitals have responded to the last

\section{Methods and results}

I sent a questionnaire to 100 randomly selected accident and emergency departments. Non-responders were contacted once by telephone. The questionnaire asked the identity and training of the medical incident officer; the members, training, and experience of the mobile medical team; and whether action cards were issued.

Ninety three departments returned questionnaires. Five departments were excluded from analysis because the procedures were undergoing major revision, leaving 88 for analysis.

The medical incident officer was a member of an immediate care team in six hospitals, a hospital consultant in 80 hospitals, and a senior house officer in two. Twenty nine departments specified an accident and emergency consultant and eight a member of the hospital flying squad. Eight had chosen physicians, eight anaesthetists, five orthopaedic surgeons, 10 obstetricians or urologists, and 14 other specialists. Training was given through practical exercises in 16 departments, table top exercises in five, courses in 14 , and local immediate care schemes in seven; 46 hospitals gave no specific training.

The mobile medical team leaders included various specialties and grades (table). Only 10 teams had experience in treating patients before arrival at hospital. Four teams were trained in conjunction with the emergency services and five trained with a flying squad or immediate care scheme. In 23 cases training was not specified and 56 had no training. Action cards were issued by 66 hospitals.

\section{Comment}

The regulations state that the medical incident officer should be a "senior clinician with appropriate
Grade and specialty of mobile medical team leader

\begin{tabular}{lccccc}
\hline & $\begin{array}{c}\text { Consultant/ } \\
\text { senior } \\
\text { registrar }\end{array}$ & $\begin{array}{c}\text { Registrar } \\
\text { grade }\end{array}$ & $\begin{array}{c}\text { Senior } \\
\text { house } \\
\text { officer }\end{array}$ & Other & Total \\
\hline Accident and emergency & 14 & & 8 & 22 \\
Surgery & 5 & 16 & 2 & 23 \\
Orthopaedics & 14 & 11 & 3 & & 28 \\
Gynaecology & 1 & 2 & & & 3 \\
Ear, nose, and throat & 1 & 1 & & & 1 \\
$\begin{array}{l}\text { Radiology } \\
\text { Unspecified }\end{array}$ & 3 & & & 7 & 3 \\
Immediate care scheme & & & & 7 & 7 \\
\hline Total & 38 & 30 & 13 & 88 \\
\hline
\end{tabular}

experience and training." But over half receive no training. Although courses improve theoretical knowledge, they do not improve familiarity with local equipment and staff. Only 28 incident officers took part in exercises and minor incidents despite many departments accepting that minor incidents provide training for major disasters. ${ }^{2}$

Local immediate care doctors who are familiar with the prehospital environment may be more appropriate for medical incident officers than hospital consultants, who may be unaware of the capabilities of paramedics and the local acute facilities.

It is encouraging that a consultant or senior registrar led the mobile medical team in 38 hospitals, and that 72 included trauma specialists. Whether senior house officers should be team leaders is questionable. Unfortunately, the guidelines do not differentiate between a resuscitation team and a surgical team.

Familiarity with the cramped conditions, bad weather, and the limitations of care outside hospital are more important than specialty or grade. But only 10 teams had any experience at accident sites. Redmond suggested using regional teams so that someone had previous experience of a major incident. ${ }^{3}$ Local immediate care teams are ignored in most major incident plans.

Arrangements for on site medical services remain inadequate in most hospitals. There seems to be a lack of specific training. Hospitals should consider whether it is more suitable to contract their major incident on site services to a specialist trained group.

1 Department of Health. Emergency planning in the NHS: health services arrangements for dealing with major incidents. London: HMSO, 1990. ( $\mathrm{HC}(90) 25$.)

2 Fisher J. The British association for immediate care. Its experience in major disasters, with special reference to the role of the medical incident officer. Injury 1990;21:45-8.

3 Redmond AD. Disaster management. BMF 1989;298:962.

(Accepted 7 fuly 1992)
WHO Collaborating Centre for International Drug

Monitoring, Box 26, S-75103 Uppsala, Sweden Sten Olsson, senior pharmaceutical officer I Ralph Edwards, medical director

Correspondence to: Mr Olsson.

BMF 1992;305:748-9

\section{Tachycardia during cisapride treatment}

\author{
Sten Olsson, I Ralph Edwards
}

Cisapride is a gastrointestinal prokinetic agent that stimulates motility throughout the gastrointestinal tract. It is used for symptomatic treatment of dyspepsia and delayed gastric emptying. The pharmacological basis of its actions is not completely clear, but it probably facilitates acetylcholine release in the myenteric plexus of the gut.' Unlike the chemically related agent metoclopramide, cisapride exerts no antidopaminergic or direct cholinergic effect.

Cisapride is generally claimed to have no effect on cardiac rhythm. ${ }^{12}$ The product monographs for cisapride do not include any notice of such an effect in any of the major compendia. However, over the three years from 1989 to 1991 seven reports were submitted to the WHO Programme for International Drug Monitoring in which cisapride was associated with disturbances in heart rhythm. Reports were submitted by the national drug monitoring centres in Australia, Belgium, the Netherlands, and the United Kingdom.

\section{Case reports}

Of the seven reports received of cardiac effects related to cisapride, four were of palpitations (with associated chest and arm pain in two patients), one was of tachycardia and hypertension, and two were of extrasystoles (in one (case 3 ) supraventricular) (table). As may be seen from the table, the male to female ratio was 3:4. Patients' ages ranged from 26 to 69 years, with a mean of 52 . The administered dose of cisapride was 